\title{
Follow-the-Leader Control Strategy for Azimuth Propulsion System on Surface Vessels
}

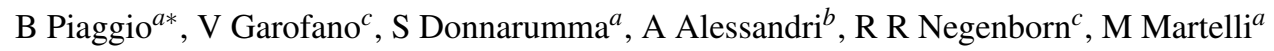 \\ ${ }^{a}$ Dept. of Naval Architecture and Marine, Electrical, Electronic, Telecommunications Engineering (DITEN), \\ Polytechnic School of Genoa University, Genova, Italy; \\ ${ }^{b}$ Dept. of Mechanical, Power, Industrial and Transport Engineering (DIME), Polytechnic School of Genoa \\ University, Genova, Italy; \\ ${ }^{c}$ Department of Maritime and Transport Technology, Delft University of Technology, Delft, The Netherlands \\ *Corresponding author. Email: benedetto.piaggio@edu.unige.it
}

\section{Synopsis}

This paper presents an effective autonomous follow-the-leader strategy for Azimuthal Stern Drive vessels. The control logic has been investigated from a theoretical point of view. A line-of-sight algorithm is exploited to ensure yaw-check ability, while a speed-check feature is implemented to track the velocity of the target along the path. For this purpose, a linearised manoeuvrability model for azimuthal drive surface vessels is presented. A model-based control synthesis is proposed to ensure the stability of the closed-loop system and robust PID controllers are designed by using Linear Matrix Inequalities technique. The control strategy has been successively validated in two steps, initially by using simulation techniques, and then experimentally using an outdoor scenario with model scale tugs.

The path planning, navigation, guidance and control modules are studied, detailed, and digitally implemented on-board of the model scale tugs. The models are supplied with GNSS+INS navigation system. Low-level management and control of Azimuthals angles and shaft revolutions is implemented on-board. High-level decentralised path planning, guidance, and control sequence evaluation are dealt with at a remote ground station.

In particular, the presented follow-the-leader strategy meets the most generic needs of platooning convoys, and, in the specific instance, of Escort convoy tugs. The operative profile of the latter concerns long-lasting and routine chases with the continuous demand of tuning heading and speed to track the target vessels, until the rare occurrence of an emergency event. In a realistic scenario, the proposed control system would be beneficial for the tug master's lucidity and alertness, while reducing avoidable risks.

At the end of the paper, the results of the experimental campaign are shown to demonstrate the effectiveness of the proposed control logic.

Keywords: Follow-the-Leader, Tugs, Azimuthal Propulsion, Line-of-Sight, Path-Following, Platooning

प

\section{Introduction}

The art of navigation is one of the most crucial tasks each bridge officer should facing with. Nowadays with the development of the technologies, the design of a control system in guaranteeing the highest quality and reliability becomes imperative and creates several challenges for the scientists.

The most of the operative life of a sailing vessel itself concerns the ability to track in route and speed a predetermined path, basically in open waters and oceans; however, the capability of forming convoys and following

\section{Authors' Biographies}

Dr. Benedetto Piaggio achieved the PhD degree in Naval Architecture and Marine Engineering at the University of Genoa in 2020 , where currently is assistant researcher. In 2019 spent a visiting research period at Delft University of Technology university - ResearchLab Autonomous Shipping (RAS). His research field embraces surface vessels' hydrodynamic modelling, manoeuvrability simulation and navigation control. Amongst his main interests, tug manoeuvrability and towing cooperative dynamics are strongly concerned.

MEng. Vittorio Garofano graduated in Mechatronic Engineering MSc in 2015 at the Polytechnic University of Turin. Currently he is a Research Support Engineer in the Section of Transport Engineering and Logistic at Delft University of Technology, and, since 2019, the technical developer of the research projects carried out in the ResearchLab Autonomous Shipping (RAS). His main research topics are applied control theory, adaptive and robust control, formation and multi agent control algorithms, real-time embedded software for autonomous ship system.

Dr. Silvia Donnarumma received the Ph.D. degree in mathematical engineering and simulation from the University of Genoa, Genoa, Italy, in 2016. Her research interests include the study and application of nonlinear control techniques, based on hybrid approaches (with resets) and on convex optimization techniques based on LMIs, for the synthesis of feedback control systems; dynamic positioning system; control with actuator saturation, and automatic steering in general.

Prof. Angelo Alessandri received the Ph.D. degree in electronics engineering and computer science from the University of Genoa, Genoa, Italy in 1996. After a period as CNR research scientist, in 2005 he joined the University of Genoa, where he is currently an Associate Professor. His main research interests include moving-horizon estimation, state observers, and optimal control.

Prof. R.R. Negenborn is Full Professor on Multi-Machine Operations \& Logistics, Head of Section Transport Engineering \& Logistics at Delft University of Technology. His research interests include automatic control and coordination of transport technology (including autonomous vessels). His research presented in over 200 peer reviewed publications anticipates the massive introduction of sensing, computation, and communication technologies.

Prof. Michele Martelli received the Ph.D. degree in marine engineering and naval architecture in 2013. Currently, he is Associate Professor at the University of Genova. He is a co-author of more than 50 scientific papers. His main research interest focuses on the study of the dynamics of the propulsion plant and its control system. Since 2010 , he has been actively working on several research projects, funded by both private and public companies. 
a leading target-vessel within its wake becomes a valuable feature in both blue seas and busy/restricted areas. In the last scenario, the two main challenges are to steer and keep off-track tolerances to a minimum threshold and to tune speed accordingly to the leader, while keeping a safe distance.

In this instance, this paper presents an effective autonomous follow-the-leader strategy valid in general for Azimuth-Drive surface vessels. The azimuth-driven class of vessels is connotated by high and flexible $0-360^{\circ}$ thrust allocation features and manoeuvring capabilities. These qualities turn to be ideal for both work-vessels such as tugs and Offshore Supply Vessels (OSV) (z-drive ducted propellers), and merchant vessels (ducted or poddedpropellers) that approach to restricted/inland areas and harbours without the need of external assistance. The class of azimuth-driven vessels indeed represents the most competitive solution in handling for steering vessels both at low and high-speed, and thus moved the prime interest of the authors.

The ability to follow a moving target along its route meets the most restrictive needs of sailing in crowed and restricted areas, such as harbours and channels, where traffic management is crucial (Schiaretti et al., 2017a b). The narrower the navigation lane, the greater the demand of precision of the guidance, the navigation and the control (GNC) system. This dictates the requirement of gathering vessels in assembled and neat "train" convoys in compliance with the traffic control rules, i.e. multi-body vessel platoons. In particular, especially this theme is believed by the authors to be a crucial step towards autonomous shipping in the EU social perspective, and thus moved the rationale of the study.

Several works in the literature deal with the theoretical idealisation and design of aided-navigation follow-theleader control systems (Caccia et al., 2008; Encarnação and Pascoal, 2001, Ghommam and Saad, 2018; Pettersen and Nijmeijer, 1998; Breivik et al., 2008, Xie et al., 2018, Bibuli et al., 2012; Chen et al., 2019, 2018, Chen et al. 2019), but most of them remain simulation-based, and not fully-contextualized with real vessel dynamics and modelling. Amongst them, many parties work with experimental demonstration of the trustworthiness of the algorithms (Schiaretti et al., 2017b) but, in any case, only few presented results for follow-the-leader techniques (Bibuli et al., 2009; Zhang et al., 2019, Belleter et al., 2019). In particular, almost no model experiments in an outdoor GNSS-INS based navigation systems exist. Switching from simulation to real testing indeed is a great challenge. A reliable simulation environment certainly is strategic for model-based control design purposes (Martelli et al., 2019, Alessandri et al.,2019), but real world always overcomes many of the ideal assumptions made to build it. The algorithms precision must deal with real-odometry complications and real system non-linearities (Donnarumma et al., 2018; Martelli and Figari, 2017). Moreover, as most of the times engineering applications suggest, the simplest the system, the most robust. The degree of sophistication of the algorithm cannot disregard the quality and precision of the whole system itself.

The novel algorithm presented in this work proposes an effective autonomous follow-the-leader strategy for Azimuthal Stern Drive vessels. The control logic is investigated from a theoretical point of view. The wellconsolidated line-of-sight algorithm (LOS) is exploited to ensure yaw-check ability, while a simple supplementary speed-check feature ( $(\mathrm{LOS}+\mathrm{V})$ is implemented to track the velocity of the target along the path (Fossen et al. 2003, Breivik and Fossen, 2004, Donnarumma et al., 2020). To this purpose - an original linearised manoeuvrability model for azimuthal drive surface vessels is deduced starting from the complete non-linear modelling proposed by Piaggio et al. (2019); Figari et al. (2020). In combination with this, a novel model-based control synthesis is proposed to ensure the stability of the closed-loop system and robust PID controllers are designed by using Linear Matrix Inequalities (LMI) techniques (Alessandri et al., 2015). Finally, the control strategy has successively been validated in two steps by Piaggio (2020), initially by using simulation techniques, and then experimentally in a unique outdoor scenario with model scale vessels. The present paper presents a brief insight into the preliminary experimental results.

The whole picture discloses an innovative and proactive control-design framework for azimuth-drive surface vessels, starting from its theoretical formulation, passing through an original LMI model-based synthesis, and finally leading to its practical and successful system implementation in the real world.

\subsection{System Architecture}

The block algebra representing the proposed Follow-the-Leader strategy is reported in figure 1 The goal of the control feature is the pursuit of the target ship with converging cross-track error and speed to the leader's path. The following vessel receives the position of the leader that is moving in front of it through the communication system, managed by the ground station. The control inputs of the following tug are the azimuth angles and the revolutions, and they are assessed by the on-board control station.

The path-planning module first collects, records, and elaborate target's trajectory on board the tug. Then, the Guidance, throughout a line-of-sight algorithm processes and translates the relative position errors into heading and speed set-points.

The module LMI PID control includes the heading and speed pilot control system, they convert heading and speed errors into actuators lever positions (azimuth angle and shaft-line revolution), which are the most suitable 


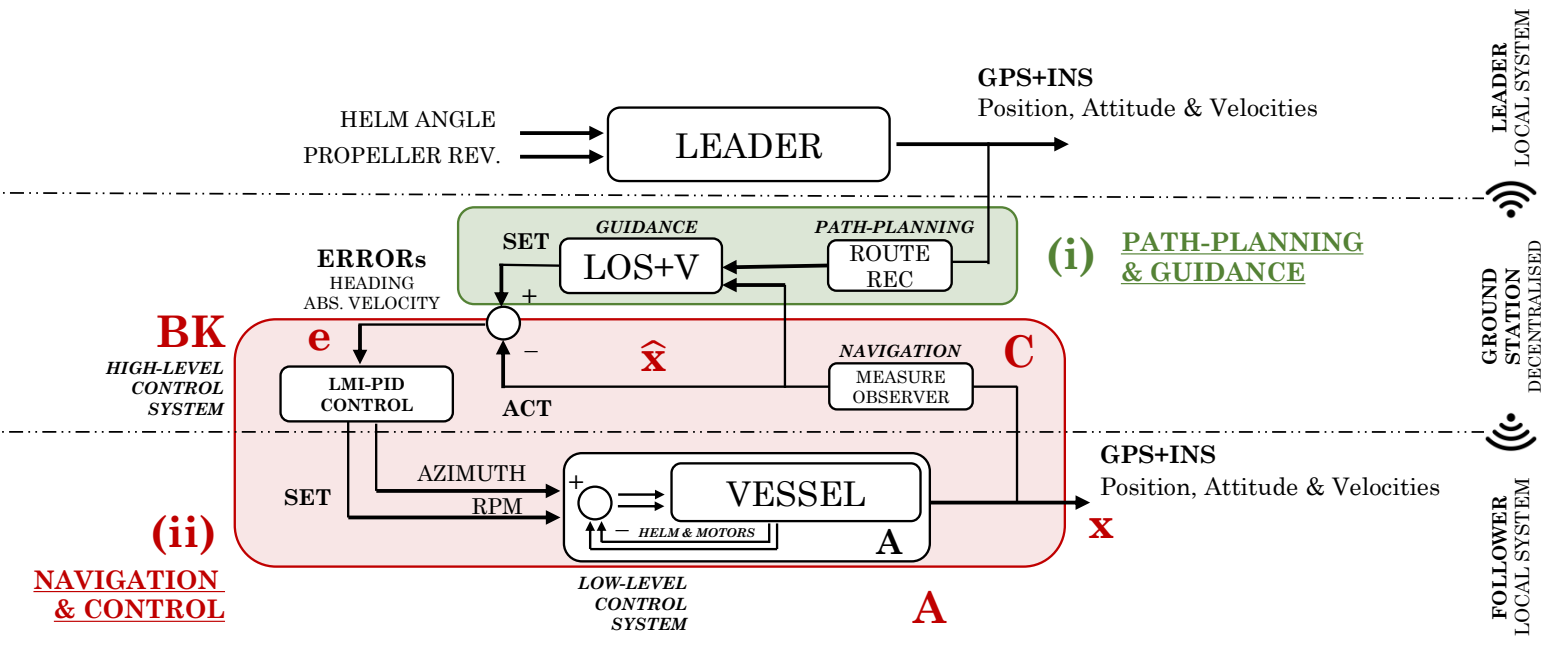

Figure 1: Follow-the-Leader scheme

control actions to comply with the path-following control goals.

The navigation module is designated to sense and observe positions, speeds and headings.

A closed-loop structure arises, based on path planning pre-processed relative positioning and speed considerations. The stability of the cascaded guidance and control modules must be ensured in each sub-part in the tug perspective. Last but not least, the measurement quality and reliability of both the navigation modules must be guaranteed.

\subsection{Experiments}

A model-scale outdoor experiment with two 1:33 Azimuthal Stern Drive tugs is presented (figure 2 - for full details see Haseltalab and Negenborn (2019)). The models, named TN3212, are equipped with an IMU unit (magnetometers, accelerometers, gyroscopes) and a GPS The models are controlled in RPMs and Azimuth angles, locally, with a low-level loop. The guidance and control of navigation are realised by means of two independent decentralised computers which close the high-level feedback loop. A communication bridge between the two broadcasts and echos the position information. A wireless serial communication protocol is adopted. Complete details can be found in the experimental setup section.

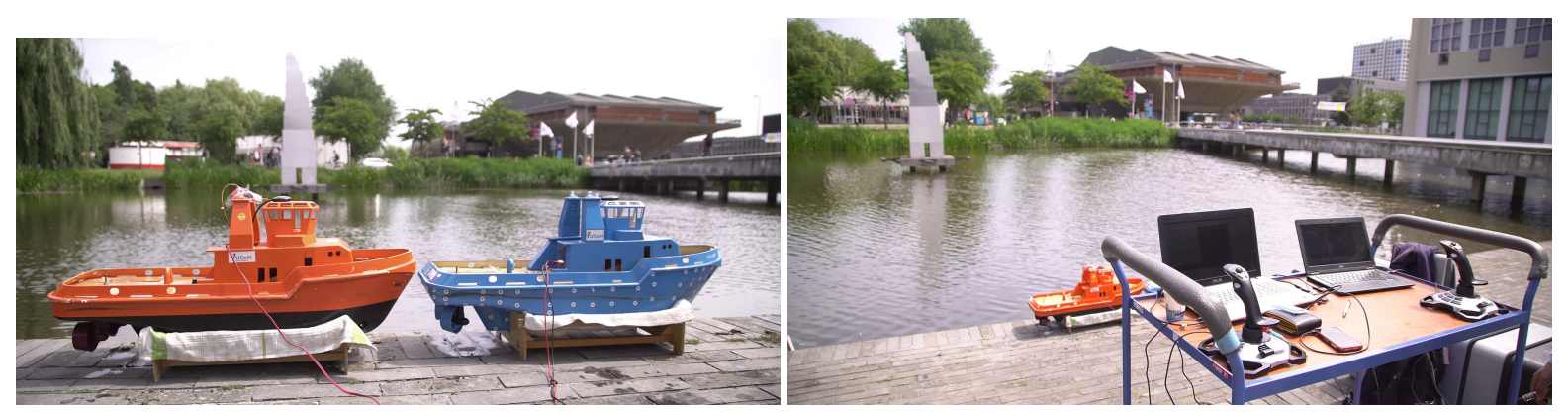

Figure 2: TN3213 models \& ground-Station

The paper is structured as follow: Section2 2 reports the theoretical insight into the Mathematical Formulation of the Follow-the-Leader strategy. The details of the Path Planning, Guidance and Control modules are supplied with the linearised vessel model used for design purposes. Moreover, in Section 2.4, the application of LMI techniques for the PID design is shown. Section 3 reports the proposed experimental setup and the manoeuvres carried out to validate the proposed strategy. Many promising results and conclusions are drawn consequently, in the Section 4

\section{Mathematical Formulation}

Figure 3 sketches the details of the problem's geometry. The path recording and filtering of the leader-vessel trajectory could be liken to the role of the classic Path Generation step. According to this, a complementary Lineof-Sight \& Speed-check algorithm [LOS+V] is used to supply suitable heading and speed set-points (Breivik and 


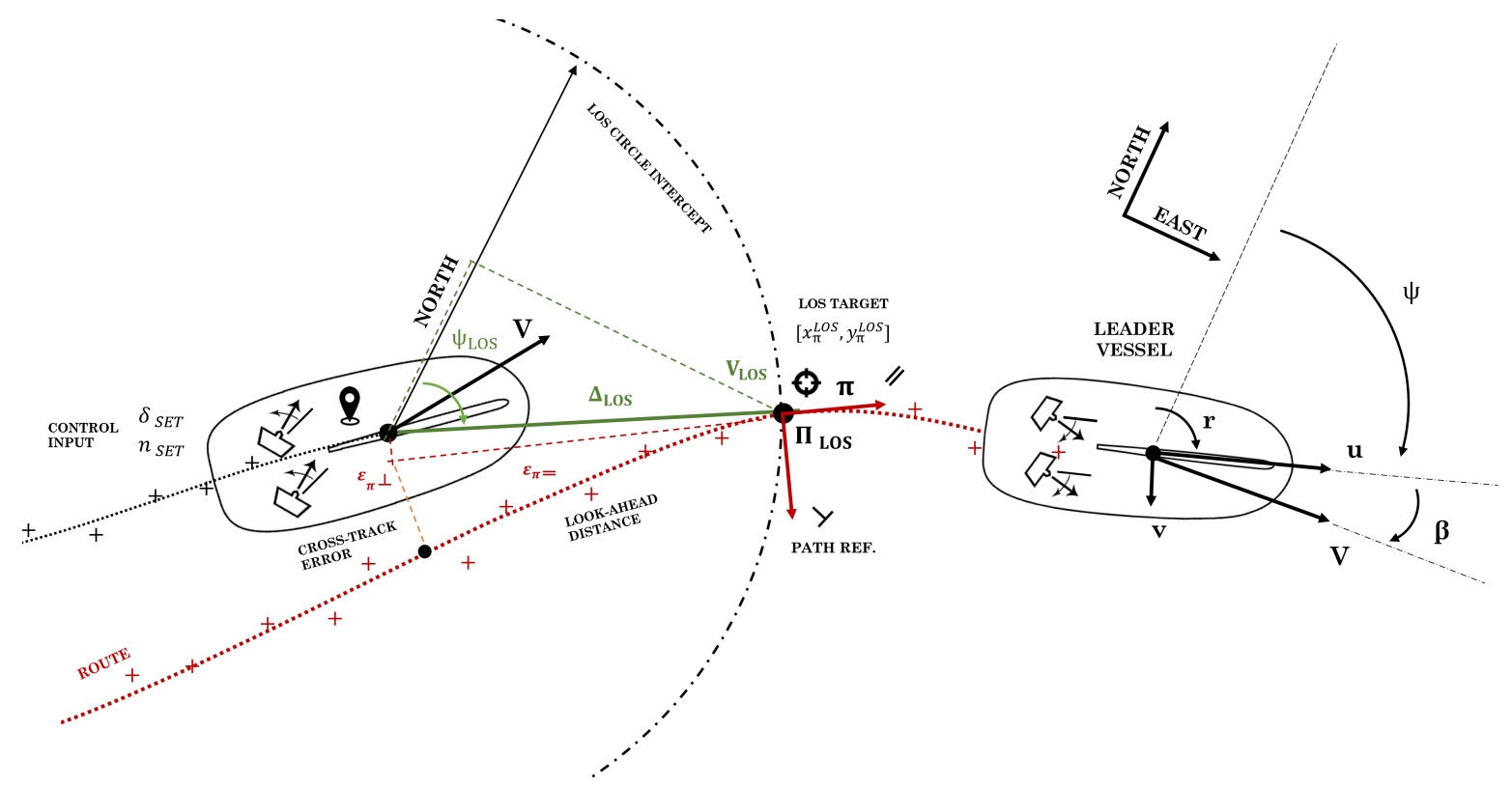

Figure 3: Geometrical overview of the algorithm LOS+V

Fossen, 2004; Fossen et al., 2003), whose aim is to ensure the geometrical convergence of the guidance module to the traced path. Then, the considered tugs's model is presented, which results from the linearisation of the non linear one detailed in (Piaggio et al., 2019, Figari et al., 2020, Piaggio, 2020), at cruise speed.

The autopilot and speed-pilot controllers are based on the azimuthal actuators, given by the helm angles and propeller revolutions. Two PID controllers are designed by relying Lyapunov arguments, according to Linear Matrix Inequality techniques (Alessandri et al., 2015). The closed-loop stability in both heading and speed is thus guaranteed. In conclusion, the overall system's stability is thus ensured in agreement with the cascadedhierarchical architecture.

\subsection{Path-Planning}

The leader's trajectory is collected and processed by the follower's path planning module in order to supply a regular and smooth trajectory to be fed to the guidance and control algorithm. A filtering process is included in the preprocessing of the leader's raw-GPS data. It has been used a Savitzky-Golay filter, which is a digital linear timeinvariant convolution filter: the principle is based on the discrete signal fit over a moving window (size $m$ samples) with a low-degree polynomial (e.g. cubic). Linear least-squares method is applied. Successive subsets of adjacent data points are analysed, and the convolution coefficients are derived through pseudo-inverse $\boldsymbol{W}=\left(\boldsymbol{H}^{T} \boldsymbol{H}\right)^{-1} \boldsymbol{H}^{T}$. As a result, the output signal is re-sampled and smoothed.

$$
\overline{\boldsymbol{x}}_{\text {target }}^{i}=\left(\boldsymbol{W} * \boldsymbol{x}_{\text {target }}\right)_{i}=\sum_{j=-\frac{m-1}{2}}^{\frac{m-1}{2}} \boldsymbol{W}_{i} \boldsymbol{x}_{\text {target }}^{j+i}
$$

One of the advantages of the method is the easy recursive implementation and the simplicity in evaluating differentials and derivatives. The smoothness of the trajectory to be followed combined with the regularity of its derivatives is beneficial for the path and set-point generation to be fed to controllers.

\subsection{Guidance System}

The guidance system is split in two parts: a line-of-sight and a speed-check module. A description of both of them is detailed in the following.

\subsubsection{Line-of-sight Algorithm}

The geometrical task of the convergence of the tug's position cross-track error $\varepsilon_{\pi \perp}$ is achieved by means of the vessel's heading-check. The LOS guidance algorithm generates a sequence of LOS angles $\psi_{L O S}$, toward which the ship must head to succeed in the pursuit. The forward intercepting LOS point allows to find the latter angle $\boldsymbol{x}_{\pi}^{L O S}$ 
of the look-ahead circle of radius $\Delta_{L O S}=n L$ centred onto the tug $\boldsymbol{x}_{t u g}$ with the smoothed trajectory traced by the target $\boldsymbol{\pi}(t)$ :

$$
\left\{\begin{array}{l}
\left\|\boldsymbol{x}_{\pi}^{L O S}-\boldsymbol{x}_{t u g}\right\|=\Delta_{L O S} \\
\frac{d \boldsymbol{\pi}}{d t}\left(\boldsymbol{x}_{\pi}^{L O S}\right) \cdot \boldsymbol{\Delta}_{L O S}>0
\end{array}\right.
$$

The LOS intercept is selected such that the dot product in between the LOS vector $\Delta_{L O S}=\boldsymbol{x}_{\pi}^{L O S}-\boldsymbol{x}_{t u g}$ and the path tangent velocity vector $\dot{\boldsymbol{\pi}}=d \boldsymbol{\pi} / d t$ is strictly positive, i.e. is the one guaranteeing an advance speed contribution in the direction of the target displacement. Thus, LOS intercept angles $\psi_{L O S}$ and local tangent to the intercepted path $\gamma_{L O S}$ can be easily derived, exploiting simple trigonometric considerations:

$$
\begin{array}{r}
\psi_{L O S}=\tan ^{-1} \frac{y_{\pi}^{L O S}-y_{t u g}}{x_{\pi}^{L O S}-x_{t u g}} \\
\gamma_{L O S}=\tan ^{-1} \frac{\dot{y}_{\pi}^{L O S}}{\dot{x}_{\pi}^{L O S}}
\end{array}
$$

\subsubsection{Speed-check Algorithm}

During the path following task, the vessel is subject to the dynamic requirement of adjusting and conforming its speed to the target, i.e. the transit velocity along the path traced by the leader vessel $V_{\pi}(t)=\|\dot{\boldsymbol{\pi}}\|$. This is driven by the LOS intercept local path speed in the time parametrisation $V_{\pi}^{L O S}(t)=\left\|\dot{\boldsymbol{\pi}}\left(\boldsymbol{x}_{\pi L O S}\right)\right\|$, plus an additional cruise control input action $d V_{c c}$, which enables the system to get nearer or further to the target:

$$
V_{t u g} \underset{t \rightarrow \infty}{\longrightarrow}\left\|\dot{\boldsymbol{\pi}}\left(\boldsymbol{x}_{\pi L O S}\right)\right\|+d V_{c c}
$$

\subsection{Vessel's Dynamics}

Starting from Piaggio et al. (2019, 2020); Piaggio (2020), a 3-DoF linearised version of the tug dynamics is derived around the straight-ahead sailing point at design speed $u_{d}$ for the present control-design scope. The hydrodynamic modelling of the vessel distinguishes the contributions in two main parts: $H$ and $A Z$ superscripts denote hull and azimuthal contributions, respectively. Jacobians of both the hull, azimuthal, and interaction parts are exploited in terms of kinematics, i.e. velocities $\boldsymbol{v}=[u, v, r]^{T}$, its derivatives, i.e. accelerations $\dot{\boldsymbol{v}}$, and its control actions $\boldsymbol{u}=[n, \delta]^{T}$. The position of the vessel in the earth-reference $\eta=[x, y, \psi]^{T}$ can be obtained by means of the planar rotation of the body-fixed velocities $\boldsymbol{v}$. Complete equations are thus deduced in concise matricial form:

$$
\begin{aligned}
\left(\boldsymbol{M}+\boldsymbol{M}_{A}\right) \dot{\boldsymbol{v}}+\overline{\boldsymbol{C}} \boldsymbol{v}-\overline{\boldsymbol{D}} \boldsymbol{v} & =\overline{\boldsymbol{\tau}}_{A Z}[\boldsymbol{n}, \boldsymbol{\delta}]^{T} \\
\dot{\boldsymbol{\eta}} & =\boldsymbol{R}(\psi) \boldsymbol{v}
\end{aligned}
$$

where $\overline{\boldsymbol{C}}$ represents the linearised Coriolis-centripetal matrix at the design speed, and $\overline{\boldsymbol{D}}$ summarises the linearised passive damping matrix in which the hull's and azimuthal's passive hydrodynamic contributions merge; $\boldsymbol{R}(\psi)$ is the rotational matrix; $\boldsymbol{M}+\boldsymbol{M}_{A}$ represents the mass and added-mass matrices.

The active part of the azimuthals is retained in the actuator forces array $\overline{\boldsymbol{\tau}}_{A Z}$. In detail, the latter counts the portside and starboard contributions. This plays the role of the control input array $\boldsymbol{u}$ in terms of the azimuth angles and revolutions, where $\boldsymbol{u}=[n, \boldsymbol{\delta}]^{T}$.

In the linearised context (small variations of the variables), propeller revolution, and azimuth angle effect play separated roles in surge and sway+yaw dynamics, de facto uncoupling completely the problem in two subproblems. Moreover, port-side and starboard-side azimuthals contributions become identical, leading to a factor 1 or 2 in case they are used individually or twin.

At the end, actuation dynamics are included and simplified through two additional first-order differential equations. Time constants representative of the response of azimuthal's revolution raise $\tau_{A Z}^{n}$ and helm angle settling $\tau_{A Z}^{\delta}$ are thus introduced to model mechanical/control limits to systems' step responses to revolution $n_{S E T}$ and helm angle $\delta_{S E T}$ setpoints.

$$
\begin{aligned}
& \dot{n}=1 / \tau_{A Z}^{n}\left(n_{\text {set }}-n\right) \\
& \dot{\delta}=1 / \tau_{A Z}^{\delta}\left(\delta_{\text {set }}-\delta\right)
\end{aligned}
$$




\subsection{LMI-PID Control System}

\subsubsection{PID Controllers}

The maritime industrial practice fosters the simplicity and robustness of control algorithms (Donnarumma et al. 2018; Martelli and Figari, 2017). PID structures indeed enable to control successfully most of the mechanical systems, which are reducible to second-order dynamics, by means of suitable zeros and poles placement techniques in the frequency-domain. The control is achieved by evaluating the actual error of the desired quantity to be controlled and by devising a suitable control action. Three coefficients are adopted for the tuning, i.e., the proportional $k_{p}$, integral $k_{i}$, and derivative $k_{p}$ parameters. The transform function to be dealt is $G(s)=\frac{k_{d} s^{2}+k_{p} s+k_{i}}{s}$. Speed $e_{u}:=\left(u_{r}-u\right)$ and heading $e_{\psi}:=\left(\psi_{r}-\psi\right)$ error dynamics in closed-loop are given by:

$$
\begin{aligned}
& n_{\text {set }}=k_{p}^{u} e_{u}+k_{i}^{u} \int_{0}^{t} e_{u}(\tau) d \tau+k_{d}^{u} \dot{e}_{u} \\
& \delta_{\text {set }}=k_{p}^{\psi} e_{\psi}+k_{i}^{\psi} \int_{0}^{t} e_{\psi}(\tau) d \tau+k_{d}^{\psi} \dot{e}_{\psi}
\end{aligned}
$$

which reads the necessity of inclusion of two new state variables $\dot{\varepsilon}_{u}:=\int_{0}^{t} e_{u}(\tau) d \tau$ and $\dot{\varepsilon}_{\psi}:=\int_{0}^{t} e_{\psi}(\tau) d \tau$, describing the dynamics of the integral errors during the system's evolution.

\subsubsection{LMI Controller Synthesis}

The present section proposes the control synthesis technique based on Lyapunov stability of linear systems (Alessandri et al., 2015). The modelled architecture lays the ground on the augmented linear system described by the set of tug's dynamics and kinematics (Eq. 5), the actuation dynamics (Eq. 6) and the control dynamics augmented of the error dynamics (Eq. 7). Let $\boldsymbol{x}=\left[u, v, r, \psi, n, \delta, \varepsilon_{u}, \varepsilon_{\psi}\right]^{T}$ be the augmented state of the system, and $\boldsymbol{A}$ its resulting augmented transition matrix. The resulting linear matrix description of the system is thus so derived by means of the state feedback control action $\boldsymbol{u}=-\boldsymbol{K} \boldsymbol{x}$, where the matrix $\boldsymbol{K}$ collects the PID parameters. Explicit complete closed-loop dynamics are thus obtained:

$$
\dot{\boldsymbol{x}}=(\boldsymbol{A}-\boldsymbol{B} \boldsymbol{K}) \boldsymbol{x}+\boldsymbol{E} \boldsymbol{u}_{\text {set }}
$$

The aim of the controller design is the evaluation and shaping of the stability properties of the $(\boldsymbol{A}-\boldsymbol{B K})$ closedloop matrix up to the shifting part $\boldsymbol{E} \boldsymbol{u}_{\text {set }}$. Lyapunov stability criteria concern the individuation of a matrix $\boldsymbol{Q}>0$ such that, for $x \neq 0$ :

$$
\begin{array}{r}
V(\boldsymbol{x})=\boldsymbol{x}^{T} \boldsymbol{Q x}>0 \\
\dot{V}(\boldsymbol{x})=\dot{\boldsymbol{x}}^{T} \boldsymbol{Q} \boldsymbol{x}+\boldsymbol{x}^{T} \boldsymbol{Q} \dot{\boldsymbol{x}}<0
\end{array}
$$

Given the state dynamics - which depends on the state-feedback control matrix $\boldsymbol{K}$ - the problem is laid down in the two matrices unknowns $\boldsymbol{Q}$ and $\boldsymbol{K}$. The system is quadratically stabilisable if this pair is such that:

$$
\begin{aligned}
\boldsymbol{Q} & >0 \\
(\boldsymbol{A}-\boldsymbol{B} \boldsymbol{K})^{T} \boldsymbol{Q}+\boldsymbol{Q}(\boldsymbol{A}-\boldsymbol{B} \boldsymbol{K}) & <0
\end{aligned}
$$

Equivalently, the same blinear expressions are converted to a linear problem, by pre and post-multiplying for $Q^{-1}$, and switching variables to the pair $\boldsymbol{P}:=Q^{-1}$ and $\boldsymbol{Y}:=K \boldsymbol{Q}^{-1}$. This results in the more convenient LMI form given by:

$$
\boldsymbol{P} \boldsymbol{A}^{T}-\boldsymbol{Y}^{T} \boldsymbol{B}^{T}+\boldsymbol{A P}-\boldsymbol{B} \boldsymbol{Y}<0
$$

The proportional, derivative and integral control gain is thus deduced once the problem is solved, reversing back to the original variables, i.e. $\boldsymbol{K}=\boldsymbol{P}^{-1} \boldsymbol{Y}$.

\section{Experimental Validation}

\subsection{Set-up \& Facilities}

In order to validate the previous controller synthesis, an experimental campaign is designed and carried out. Two 1:33 TN3212 model-scale Azimuthal Stern Drive tugs (Fig. 2) were instrumented for outdoor remotecontrolled navigation (Fig. 4). 
The TN3212 manoeuvring linear coefficients were deduced and calibrated according to a customary identification optimisation process in the first steps of the experimental activities, based on Piaggio et al. (2019); Piaggio (2020). A sketch of the experimental set-up scheme is reported in figure Fig. 5. Each vessel was equipped with two distinct Arduino Mega 2560 micro-processors MCU, the "Main" and the "Navigation." The former is responsible for the low-level control and communication; the latter manages the sensor's data.

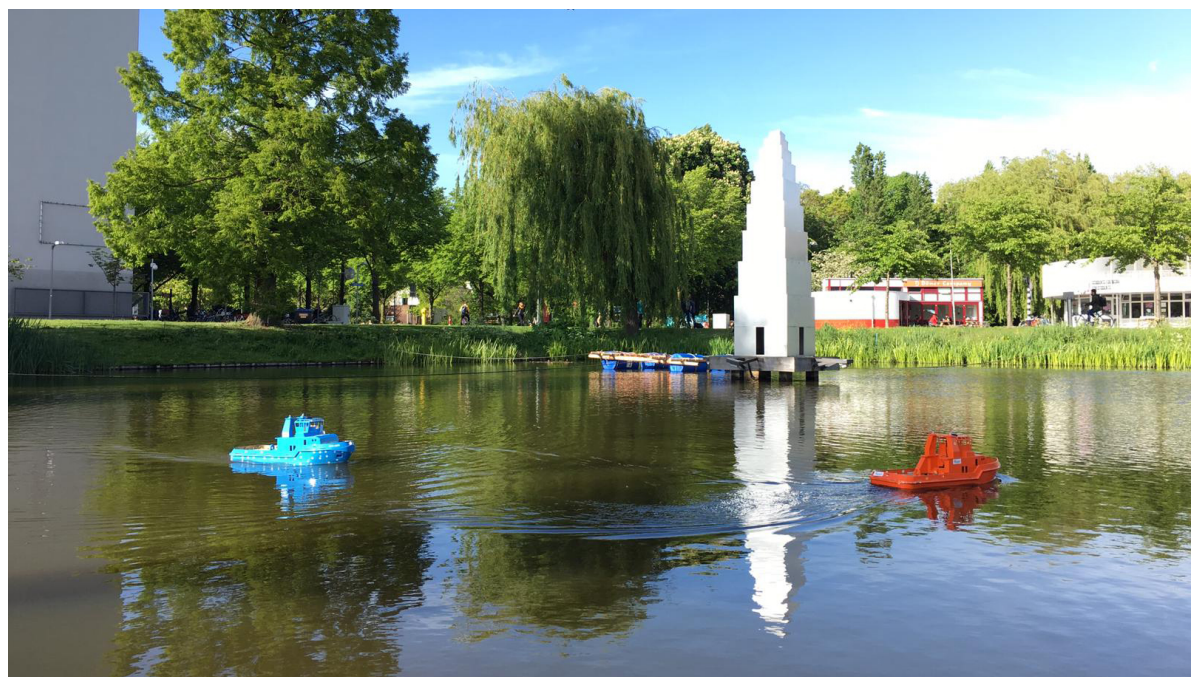

Figure 4: Experimental model-tests

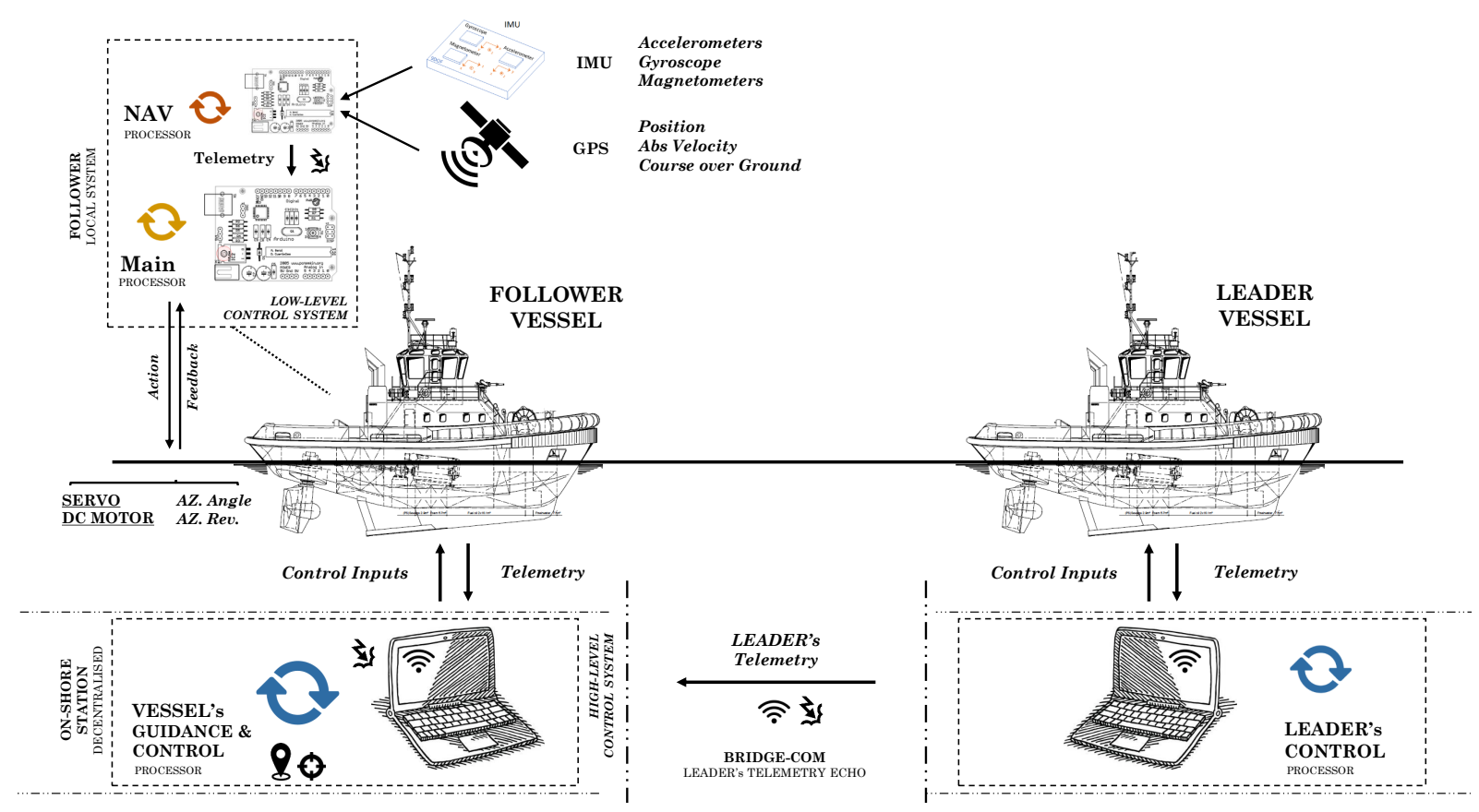

Figure 5: Experimental set-up

The Main Processor was intended as the local on-board low-level controller of azimuthals in angles and revolutions and the querier and collector of the sensed telemetries. The internal clock rate was shaped on the engine's shaft revolution frequencies so that it was possible to manage its control. Engines' maximum rates are about 3300 RPMs. The management of the PWMDC-engine powering in revolution and the helm servo in angle are internally feedback loop controlled.

The Navigation Processor was devised for gathering the freshest onboard navigation instrumentation data at $100 \mathrm{HZ}$ sampling frequencies, ready to supply it to the Main at any query. A geo-reference GPS receiver (Ublox 
Neo-7m - $1 \mathrm{~Hz}$ ) and an inertial 9-DoF IMU sensor (Adafruit BNO055 - $100 \mathrm{~Hz}$ ) were installed onboard. Interrupts and callbacks were exploited with such an aim. Serial communication is supplied to connect to the Main processor directly.

On-shore Control Stations Decentralised high-level control was conceived through two independent laptops, connected by means of wireless Xbee asynchronous serial communication to the vessels. At PC's query the vessels' Mains forward the telemetries, the computer elaborates and replies to the vessel with control inputs. Path-Planning and Guidance were thus remotely processed and overlaid onto the screen in a user interface, while control actions were sent back to the vessels, in this way closing the high-level feedback loop. A high-level $10 \mathrm{~Hz}$ control loop frequency was guaranteed to appreciate model scale dynamics and kinematics useful for guidance and control together with the INS refresh, while GNSS updates run at $1 \mathrm{~Hz}$.

Bridge Communication A second wireless Xbee asynchronous serial cross-communication between the laptops was set-up in order to share relative positioning information. Leadership telemetry was constantly shared to the trailing tug by means of another callback, triggering at each data refresh. In case of delay in refreshing or communicating new data, the tug proceeds with previous information and estimates without crashing. The two tugs were thus conceived completely independent and robust to each other failure and in case of emergency fully recoverable in real-time manual mode.

Experiments were carried out at TUDelft facility lake, which is about $25 \times 25 \mathrm{~m}$ sized (Fig. 4). Manual control through a joystick was provided to start navigation and backup in case of failures to recover the vessels. The Follow-the-Leader strategy was activated, once the vessels were initialized at speed. The integral part was smoothly activated to avoid initial bump cumulative errors. No tow line was secured in order to check the best suitability of the controllers without its aid. Azimuthals were helmed parallel and tuned synchronously in engine revolutions. The maximum angle was selected about $45 \mathrm{deg}$, while max engine revolutions were about $3300 \mathrm{RPM}$. Experiments have been conducted with a sailing speed such as in full-scale correspond to a ranges of 6-12 knots. The orange tug is the leader, manually or automatically sailing with varying course and speed, while the blue tug autonomously follows (see Fig. 4).

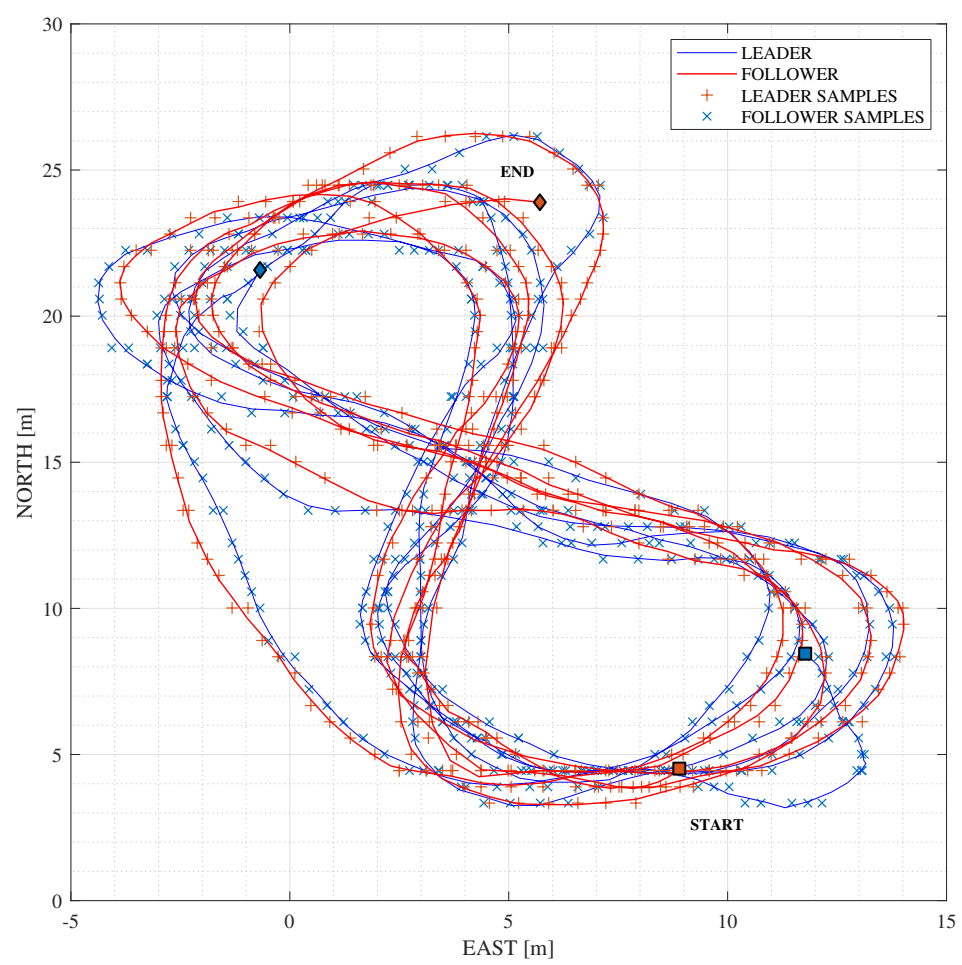

(i) Convoy Trajectories

Figure 6: Experimental model testing - 8-Runs

\subsection{Results}

The proposed validation manoeuvring scenarios concerns an 8-shaped pattern of tight turnings with varying speed, where the leader vessel was sailed manually, while the follower engaged the pursuit. 

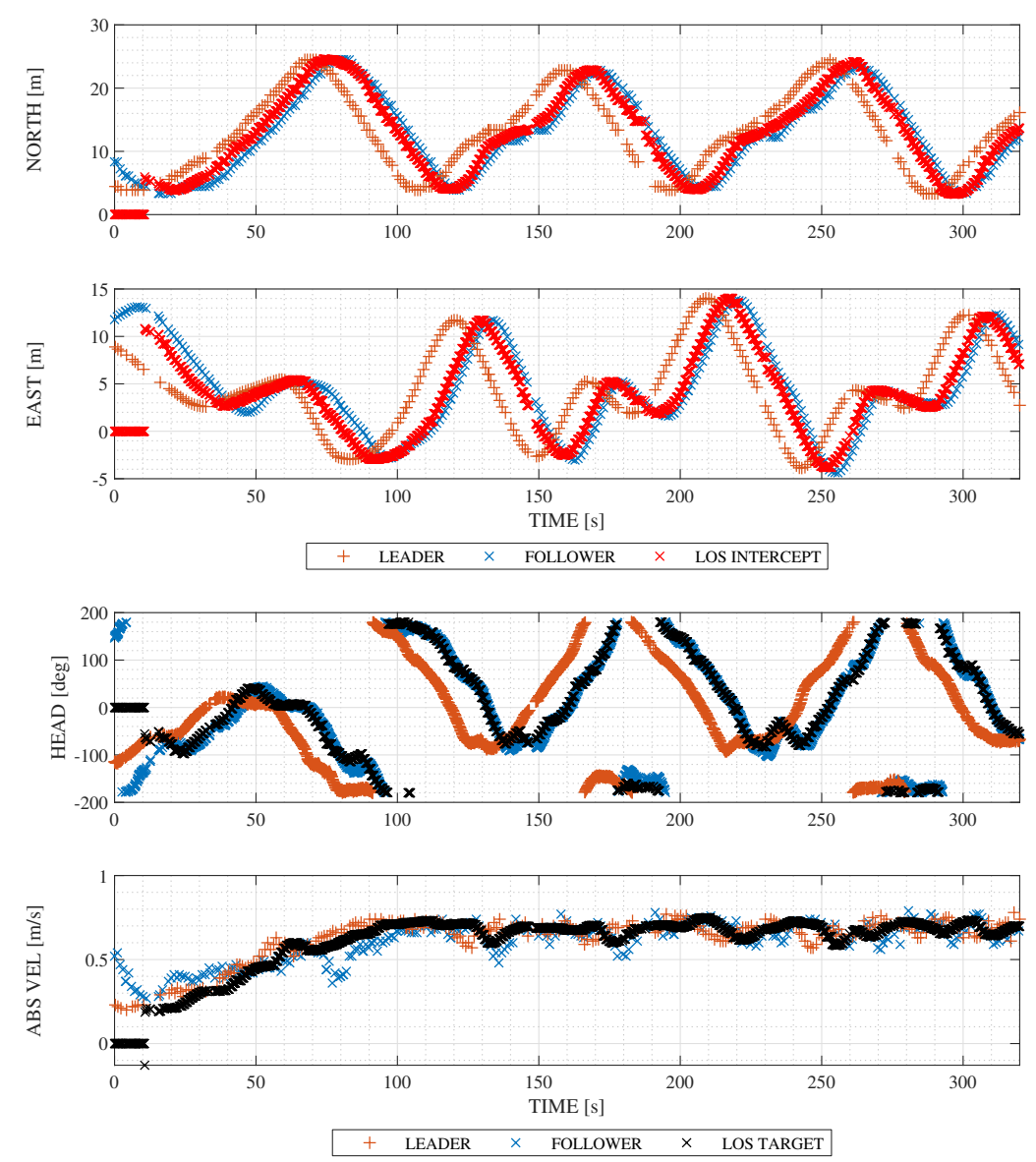

(ii) LOS+V Algorithms
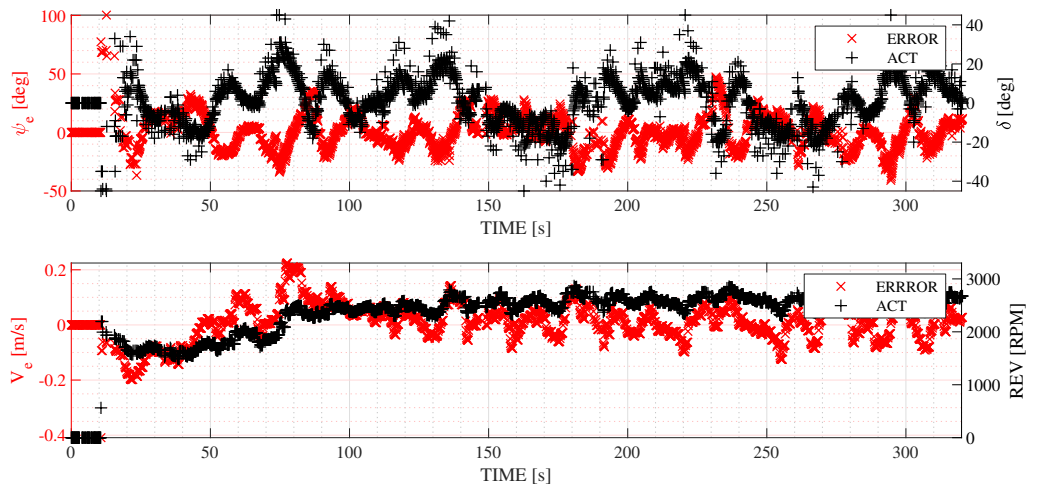

(iii) Az. Control \& Ref. Errors

Figure 7: Experimental model tsting - 8-Runs controllers

The convoy trajectories are depicted in Fig. 6(i). The orange tug is the leader (orange lines and markers), while the blue one follows (blue lines and markers). Crossed markers refer to the raw measurements of positions, processed then by the Path-Planning algorithm, which results in the solid smoothing lines. The squared and diamond markers point the start and the end of the tests, respectively. The convergence of the system in position is immediately geometrically noticeable by keeping bounded the cross-track errors within $0.3 \mathrm{~m}$ in average: the cluster of trajectories positively narrow tightens, especially with in mind the normal GPS limitations at very small scales. The presented complete test successfully lasted 10 minutes without human intervention.

For the reader's convenience, an extract of the first $300 \mathrm{~s}$ of the test is reported in the following rows. The LOS algorithm intercept coordinates (north and east) and its descending heading and speed guidance set-points are overlaid to the actual quantities in Fig. 7(ii) time-series. Controllers are started as soon as the path-planning algorithm is pre-filled and initialised within the first $10 \mathrm{~s}$. Once the target is engaged, control set-points and actual quantities almost result in a time-delayed version of the leader's state at regime up to the self-induced control 
oscillations during transient phases. In fact, the follower's series (blue marks) responsively converge in position to the LOS target intercepts values at algorithm's engagement (red marks), efficiently reacting at the leader's heading and speed variations (see actual values - blue marks - overlapping to set-points - black marks). This positively depicts the algorithm's ability in retracing the leader's path and speed at a safe distance, always keeping within its wake.

Fig. 7(iii) shows the vessel's control sequences, i.e. helm azimuth-angles and propeller revolutions. Error time-series in heading and speed (red, left axis) are compared with the actual azimuth control actions in angles and revolutions (black, right axis). This representation is used to emphasize the closed-loop response of the system. The initial transient phase (5-10s) in the activation of controllers is rapidly handled with a satisfactory convergent response. Then, in detail, the two controllers both track the smoothly changing and the faster step-wise switching set-points. On the one hand, faster and harsher responses are dealt with by heading-check controller due to the fast response to the action of azimuthals' helming. On the other hand, intentionally softer raise and descent in speed occur due to the slower dynamics in the surge speed. As a consequence, both heading and speed errors satisfactorily tend to zero in average.

\section{Conclusions and further research}

Follow-the-Leader techniques development - from design to testing - are relevant themes in the shipping world indeed, with a substantial social impact especially in the EU's vision. On the one hand, the subject matter deeply pervades many civil applications, for instance, the restricted waters and inland navigation, and in particular: the platooning of autonomous vessels and barges along inland corridors and the deployment of Escorting routes. On the other hand, the same permeate both the research and naval field, e.g. swarming, platooning, surveying/reconnaissance and cooperative formation of unmanned vehicles.

Under these terms, an original and straightforward Follow-the-Leader control-strategy for azimuth-drive surface vessels has been theoretically investigated, practically designed and experimentally validated in a novel outdoor GNSS+INS-based model scale campaign. This originally closes the loop of an innovative and proactive control-design framework from theory to the real world.

The yaw-check ability of the LOS algorithm and the speed-check tracking feature are experimentally verified based on a well-established theory. The algorithms are simultaneously active contemporary, ensuring convergence without negative interference. When turning, the speed loss and the heading errors steps are dealt from the controllers smoothly, demonstrating the robustness of the proposed controllers. The manoeuvring non-linearities do not undermine the effectiveness of the PID control in case of narrow turning, as it result from using the linearised model and the LMI-based controller synthesis.

The experiment satisfactorily demonstrates the efficacy and robustness of the tracking algorithms in all the complications of an experimental set-up which involve real-world non-scalable instrumentation precision and accuracy, wireless serial transmission delays, and lost ticks of two contemporary hardware and software independent vessels, and multi-machine clock synchronisation issues. The algorithm is simple, robust, and recursive at each time step to suit digital microprocessor programming and wireless XBee asynchronous serial communication in the high-level feedback loop at about $10 \mathrm{~Hz}$.

Further investigations will be carried out by accounting for environmental disturbances within the theoretical framework, in spite of the non-ideal testing conditions, which were been successfully positively managed by the proposed control setup.

In conclusion, the small scale complications given by the accuracy of the GPS system (that could be improved by using dGPS techniques) and the adoption of a simple and almost straight-scalable navigation and communication equipment satisfactorily fosters promising results for future real full-scale applications.

\section{Acknowledgement}

This research is supported by the Researchlab Autonomous Shipping of Delft University of Technology.

\section{Acronyms}

$\begin{array}{ll}\text { dGPS } & \text { Differential Global Satellite System. } \\ \text { DoF } & \text { Degrees of Freedom. } \\ \text { GNC } & \text { Guidance, Navigation and Control. } \\ \text { GNSS } & \text { Global Navigation Satellite System. } \\ \text { GPS } & \text { Global Satellite System. } \\ \text { IMU } & \text { Inertial Measurement Unit. } \\ \text { INS } & \text { Inertial Navigation System. } \\ \text { LMI } & \text { Linear Matrix Inequalities. }\end{array}$




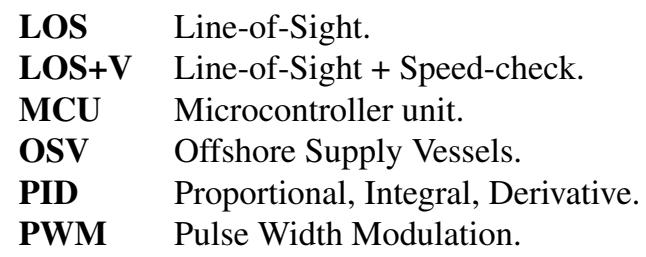

\section{References}

Alessandri, A., Donnarumma, S., Martelli, M., Vignolo, S., 2019. Motion control for autonomous navigation in blue and narrow waters using switched controllers. Journal of Marine Science and Engineering 7.

Alessandri, A., Donnarumma, S., Vignolo, S., Figari, M., Martelli, M., Chiti, R., Sebastiani, L., 2015. System control design of autopilot and speed pilot for a patrol vessel by using lmis.

Belleter, D., Braga, J., Pettersen, K., 2019. Experimental verification of a coordinated path-following strategy for underactuated marine vehicles. Frontiers in Robotics and AI 6. doi:10.3389/frobt.2019.00035.

Bibuli, M., Bruzzone, G., Caccia, M., Lapierre, L., 2009. Path-following algorithms and experiments for an unmanned surface vehicle. Journal of Field Robotics 26, 669-688. doi:10.1002/rob. 20303.

Bibuli, M., Caccia, M., Lapierre, L., Bruzzone, G., 2012. Guidance of unmanned surface vehicles: Experiments in vehicle following. IEEE Robotics Automation Magazine 19, 92-102.

Breivik, M., Fossen, T.I., 2004. Path following of straight lines and circles for marine surface vessels. IFAC Proceedings Volumes 37, 65 - 70. IFAC Conference on Computer Applications in Marine Systems - CAMS 2004, Ancona, Italy, 7-9 July 2004.

Breivik, M., Hovstein, V., Fossen, T., 2008. Ship formation control: A guided leader-follower approach. doi: 10 . 3182/20080706-5-KR-1001.3390

Caccia, M., Bibuli, M., Bono, R., Bruzzone, G., 2008. Basic navigation, guidance and control of an unmanned surface vehicle. Autonomous Robots 25, 349-365.

Chen, L., Haseltalab, A., Garofano, V., Negenborn, R.R., 2019. Eco-vtf: Fuel-efficient vessel train formations for all-electric autonomous ships, in: 2019 18th European Control Conference (ECC), pp. 2543-2550.

Chen, L., Hopman, H., Negenborn, R.R., 2018. Distributed model predictive control for vessel train formations of cooperative multi-vessel systems. Transportation Research Part C: Emerging Technologies 92, $101-118$. doi/https://doi.org/10.1016/j.trc.2018.04.013

Chen, L., Hopman, H., Negenborn, R.R., 2019. Distributed model predictive control for cooperative floating object transport with multi-vessel systems. Ocean Engineering 191, 106515. doi:https://doi.org/10.1016/ j.oceaneng.2019.106515.

Donnarumma, S., Figari, M., Martelli, M., Vignolo, S., Viviani, M., 2018. Design and validation of dynamic positioning for marine systems: A case study. IEEE Journal of Oceanic Engineering 43, 677-688.

Donnarumma, S., Figari, M., Martelli, M., Zaccone, R., 2020. Simulation of the guidance and control systems for underactuated vessels, in: Mazal, J., Fagiolini, A., Vasik, P. (Eds.), Modelling and Simulation for Autonomous Systems, Springer International Publishing. pp. 108-119.

Encarnação, P., Pascoal, A., 2001. Combined trajectory tracking and path following: An application to the coordinated control of autonomous marine craft, pp. 964-969.

Figari, M., Martinelli, L., Piaggio, B., Enoizi, L., Viviani, M., Villa, D., 2020. An all-round design-to-simulation approach of a new Z-Drive Escort Tug Class. Journal of Offshore Mechanics and Arctic Engineering 142. doi:10.1115/1.4045440.

Fossen, T.I., Breivik, M., Skjetne, R., 2003. Line-of-sight path following of underactuated marine craft. IFAC Proceedings Volumes 36, 211 - 216. 6th IFAC Conference on Manoeuvring and Control of Marine Craft (MCMC 2003), Girona, Spain, 17-19 September, 1997.

Ghommam, J., Saad, M., 2018. Adaptive leader-follower formation control of underactuated surface vessels under asymmetric range and bearing constraints. IEEE Transactions on Vehicular Technology 67, 852-865. doi:10.1109/TVT.2017.2760367

Haseltalab, A., Negenborn, R.R., 2019. Model predictive maneuvering control and energy management for all-electric autonomous ships. Applied Energy 251, 113308. doi:https://doi.org/10.1016/j. apenergy.2019.113308,

Martelli, M., Figari, M., 2017. Real-time model-based design for codlag propulsion control strategies. Ocean Engineering 141, 265 - 276. doi:https:// doi.org/10.1016/j.oceaneng.2017.06.029.

Martelli, M., Villa, D., Viviani, M., Donnarumma, S., Figari, M., 2019. The use of computational fluid dynamic technique in ship control design. Ships and Offshore Structures .

Pettersen, K., Nijmeijer, H., 1998. Tracking control of an underactuated surface vessel, pp. 4561-4566.

Piaggio, B., 2020. Azimuth-Drive Escort Tug manoeuvrability model, simulation and control. Ph.D. thesis. 
Università di Genova - XXXII Cycle in Naval Architecture and Marine Engineering Science and Technology. Via Montallegro, 16145 Genova, ITALY.

Piaggio, B., Villa, D., Viviani, M., 2020. Numerical analysis of escort tug manoeuvrability characteristics. Applied Ocean Research 97, 102075.

Piaggio, B., Viviani, M., Martelli, M., Figari, M., 2019. Z-drive escort tug manoeuvrability model and simulation. Ocean Engineering .

Schiaretti, M., Chen, L., Negenborn, R.R., 2017a. Survey on autonomous surface vessels: Part i - a new detailed definition of autonomy levels, in: Bektaş, T., Coniglio, S., Martinez-Sykora, A., Voß, S. (Eds.), Computational Logistics, Springer International Publishing, Cham. pp. 219-233.

Schiaretti, M., Chen, L., Negenborn, R.R., 2017b. Survey on autonomous surface vessels: Part ii - categorization of 60 prototypes and future applications, in: Bektaş, T., Coniglio, S., Martinez-Sykora, A., Voß, S. (Eds.), Computational Logistics, Springer International Publishing, Cham. pp. 234-252.

Xie, W., Ma, B., Fernando, T., Iu, H.H.C., 2018. A new formation control of multiple underactuated surface vessels. International Journal of Control 91, 1011-1022.

Zhang, S., Yang, S., Xiang, X., 2019. Formation control of autonomous surface vehicle and experimental validation. IFAC-PapersOnLine 52, 278 - 282. doi:https://doi.org/10.1016/j.ifacol.2019.12.421. 5th IFAC Symposium on Telematics Applications TA 2019. 